Assiut Scientific Nursing Journal

http://asnj.journals.ekb.eg

http://www.arabimpactfactor.com

\title{
Nurses and Physicians perception of Causes and Risk Ractors Contributing Patient Readmission to the Coronary Care Unit
}

\author{
Asmaa Hassanien Ahmed ${ }^{1}$, Mogedda Mohamed Mahany ${ }^{2}$, Tarek Abdelhamid Naguib $^{3} \&$ Ghada Shalaby \\ Khalaf $^{4}$. \\ 1. Teacher at secondary nursing school in Sohag city, \\ 2. Assistant Professor of Critical care and Emergency Nursing, Faculty of Nursing, Assiut University. \\ 3. Lecturer of cardiology of Assiut University Hospital, Faculty of Medicine, Assiut University \\ 4. Lecturer of Critical care and Emergency Nursing, Faculty of Nursing, Assiut University.
}

\begin{abstract}
Background: Readmission to the intensive care unit is associated with increased cost of care and worse patient outcomes Aim :of the study was to determine and explore nurses and physicians perception of causes and risk factors contributing patients readmission to coronary care unit. Design:- descriptive research design was used in this study. Setting: The study was conducted in coronary care units at a University Hospital. Sample: thirty nurses and twenty-five physicians working at the Coronary Care Unit. Tools: semi structured interview questionnaires. Results: thirty-two factors within four broad themes that could lead to readmissions were identified (1) patient factors: Previous comorbidities, Previous acute coronary syndrome, Bad medication compliance and Patient discharged before articulation goal of care (100\%). (2) process factors: Unqualified staff member, Resources constraints (100\%). (3) provider factors: Heavy workloads 90\%, Unqualified staff member deal with highly demand patient $(86,9)$. (4) organizational factors: Resources constraints (when patient transfer to ward) $100 \%$ Conclusion and recommendations: intensive care unit care givers need to look at ways of increasing the knowledge and skills of ICU staff or identify more appropriate environment for managing acutely ill patients
\end{abstract}

\section{Keywords: Readmission, Nurses, Physicians, Perception, Risk Factor, causes.}

\section{Interdiction}

Coronary artery disease is punctuated by episodes of acute exacerbation requiring hospitalization, most commonly for acute coronary syndromes (ACS). Despite improvements in acute care and survival after ACS hospitalization, early readmissions remain common and have a significant clinical and financial impact. These readmissions are seen as undesirable events both for patients and healthcare systems. (Danielle, 2014)

Readmission is defined as a second admission to the coronary care unit (CCU) during the same hospitalization. Unplanned readmissions to intensive care units (CCU) impose a significant burden on patients and the health care system. Readmission is costly and places considerable pressure on a system with finite resources. it is not surprising that research indicates that patients readmitted to $\mathrm{CCU}$ within the same hospital stay experience poor outcomes than those not readmitted. mortality rates are 6 times higher and have lengths of stay twice as long as nonreadmitted patients. They are 11 times more likely to die in a hospital. To date, only one study has examined ICU readmissions using a qualitative perspective. ( Elliot \& croocks, 2011).

Discharge plan for patients from ICU usually depends on the clinical judgment of the intensivists or on the collaboration of other members of the CCU care team. Due to the highly subjective nature of CCU discharge decisions, as well as limitations in clinical resources or an insufficient number of beds to accept all patients who need CCU care, patients can be discharged prematurely from the CCU, some of whom are inevitably readmitted. Therefore, early identification of patients at higher risk of CCU readmission would help clinicians to appropriately decide who is ready to be discharged from the CCU and prevent avoidable morbidity and mortality after discharge (Yong, 2015).

However non-patient factors such as patient inflow volumes, CCU occupancy and clinician decision making practices have also been suggested complex and error-prone care transitions between teams at the time of discharge from coronary care may also contribute to unplanned readmission. (Dongy.2017).

Proposed reasons for $\mathrm{CCU}$ readmission include recurrence of the initial complaint, complications arising from treatment, poor responsiveness to therapy, comorbid complications, onset of new medical conditions and failure of the receiving unit to cope with the level of care demanded by the patient It is not clear whether the decision to discharge patients from the $\mathrm{CCU}$ or the level of care given to these 
patients on the general wards, or a combination of both, results in readmission to the CCU. Therefore, the ability to identify patients at high risk of readmission to the $\mathrm{CCU}$ during the same hospitalization could allow objective decisions to be made by clinicians regarding the timing of discharge from coronary care, the level of care required by patients on the ward and the need for follow up by the staff. (Steven .A 2010)

\section{Significance of the study}

Readmission is costly and places considerable pressure on a system with finite resources and is an important first step in reducing patient readmission is identifying patients who are most likely to be readmitted.

Number of the patient admitted to ICU are 1250 and readmitted are 100 patients

( according to the records of the coronary care unit at Assiut university hospital 2018).

\section{Aim of the study \\ The present study aims to: \\ Identify nurses and physicians perception of causes and risk factors contributing \\ patient readmission to the coronary care unit .}

\section{Research question}

What is nurses and physician perception of causes and risk factors contributing patient readmission to the coronary care unit?

\section{Subject and method \\ Research design}

The descriptive research design was used to conduct this study.

\section{Setting}

This study was carried out in the coronary intensive care unit at Aurman Assiut University hospital that consists of 3 rooms( 16 bed), 40 nurses and 30 physicians.

\section{Sample}

A convenient sample for this study was selected and includes (30) nurses and (25) physicians working in a coronary intensive care unit at a University Hospitals. The study period was 6 months from July 2018 to December 2018.

\section{Inclusion criteria}

1- Nurses and physicians who provide direct patient care at the coronary care unit.

2- Nurses and physicians who have at least one year of experience at work.

\section{Exclusion criteria}

Newly graduated nurses and physicians and less than one year of experience.

\section{Tools of data collection}

One tool was used in this study and was designed by the researcher based on a literature review for data collection (Elliot\& croocks, 2011) .

(Semi structured interview questionnaire):-

This tool will be developed by the researcher based on review literature (Ofoma et al., 2018)

This tool aimed to assess critical care nurses and physicians perception about causes and risk factor of readmission to coronary care unit, (their awareness about risks and causes for readmission)and consists of two parts as following: .

Sociodemographic data of nurses \& physicians: ( such as code, age, sex, year, marital status, educational level, unit name, year of experience)

Nurses and physicians perception about readmission causes and risk factors which includes: four categories

(1) Patients related factors are seventeen factors (age, previous comorbidities, undefined goal of care, patient discharged on his own demand, etc. .....)

(2) Process factors are two factors as (physician patient communication and lack of communication between CCU staff and ward staff)

(3) Provider factors are eight factors (heavy work load and provider experience, etc. ....)

(4) Organizational factors as five are factors (resources constrain and institutional policies, etc. ...)

\section{Method}

Data collection phases

The study was conducted throughout two main phases, preparatory phase and implementation phase including:

(i)development of a questionnaire about causes and risk factors of readmission (two months), (ii)pilot testing of the questionnaire (one month), (iii) implementation of the questionnaire( two months).

\section{1-Preparatory phase}

1- Development of the questionnaire; the questionnaire was a tool for collecting selfreporting data where participants present their perception and knowledge about causes and risk factors of readmission to coronary care unit the questionnaire was developed from international articles and publications describing all aspect of patient readmission

2- Parts of the questionnaire

a. Questions related to demographic data (open questions)

b. Questions related to nurses and physicians perception regarding causes and risk factors of 
readmission to coronary care unit (yes or no question )

The questionnaire was designed in English because all of nurses and physicians are bachelor and master's degree and hospital documents are in English. Moreover, the participants were able to communicate in English

3- Piloting the questionnaire

The questionnaire was tested once the approval of the ethical committee was received.

\section{Pilot study}

It was conducted on 3 nurses \& 3 physicians to test the feasibility and applicability of the tools and to estimate the time needed to fill the interview. Based on the results of the pilot study, the necessary modification in the sheet was done.

The reliability test for a tool (knowledge assessment tool) by using Alpha Cronbach's test $(\mathrm{R}=0.679)$ the content validity was $(0.82)$.

4- The final the questionnaire

The last edition of the questionnaire contained thirty-two items related to causes and risk factors of patient readmission. The questionnaire included: (five questions) related to personal data (age - sex - unit name- level of experience and educational level)

5- Validity and Reliability The researcher gave the questionnaire to 6 experts in critical care nursing \& medicine to review and examine the quality of the questionnaire. Cronbach's test $(\mathrm{R}=0.679)$ with a content validity of (0.82).

1- 1-Written consent was obtained from the health teams that participated in the study, after explaining the nature and purpose of the study.

2- Health team was assured that the data of this research will not be reused without second permission.

3- Confidentiality and anonymity were assured.

4- Health team have the right to voluntary participate or refuse participation in the study without any rationale at any time.

5- The objective of the study was explained by the researcher orally to the nurses and doctors additionally to the written explanations in the covering letter of the interview.

\section{2- Implementation phase}

Once permission was granted to proceed with the proposed study, the researcher-initiated data collection. The implementation phase involved the following:

1- At the initial interview, the researcher introduced himself to initiate a line of communication, explain the nature and purpose of the study prior to answering the questions to obtain consent and cooperation from nurses and physicians.

2- The researcher interviewed each nurse and doctor individually to fill out the questionnaire sheet by nurses on duty during any shift.

3- The researcher interviewed nurses and doctors during their break time and the answers were recorded immediately.

4- Data were gathered through a semi-structured one-to-one interview. to encourage participants to speak freely. Each interview was conducted in a private office in the hospital and lasted approximately 40 minutes.

5- To ensure anonymity, confidentiality, and privacy, participants' names were not used during each interview and participants were allocated a code for the study (e.g. "nurse 1b" "nurse 2 b" etc. and "physician 1a", "2a" etc.)

6- At the start of the interviews, participants were told that the interviewer would ask about their experiences of caring for patients who had been readmitted to the CCU and use further questions to explore their responses in detail. This was done to gain a thorough understanding of contributing factors from the participant's perspective. It also provided the participant with an understanding of the interviewer's role during the interview. Each interview commenced with the same statement ("tell me your experiences of caring for patients who have been readmitted to CCU'). This consistent approach to data collection helped ensure trustworthiness. Five participants were asked to provide a detailed and honest account in response to the interviewer's questions.

7- The questionnaire sheet includes sociodemographic data, patient factors organizational factors, process factors and provider factors that are contributing to patient readmission to the coronary care unit. The total number of the questions were 55 questions for coronary care nurses and physicians.

\section{Statistical analysis}

The data were tested for normality using the Anderson-Darling test and for homogeneity variances prior to further statistical analysis. Categorical variables were described by number and percent $(\mathrm{N}$, $\%$ ), where continuous variables were described by the mean and standard deviation (Mean, SD). The t-test and ANOVA TEST were used to compare continuous variables. A two-tailed $\mathbf{p}<\mathbf{0 . 0 5}$ was considered statistically significant. All analyses were performed with the IBM SPSS 20.0 software. 


\section{Results}

\section{distrbution of demographic data}

nurses $\%$ \% doctor $\%$

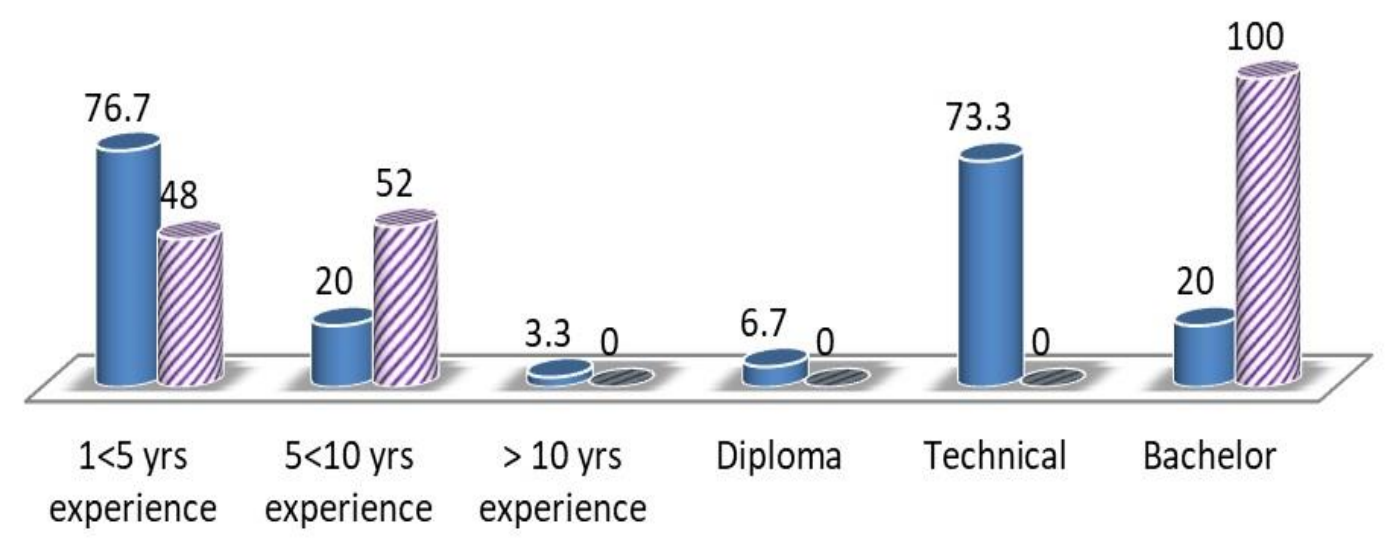

Fig (1): percentage distribution of study sample in relation to of sociodemographic characteristics data for participants ( physicians $\&$ nurses) $n=(55)$ 
Table (1): Comparison between the point of view for nurses and physician regarding patient.

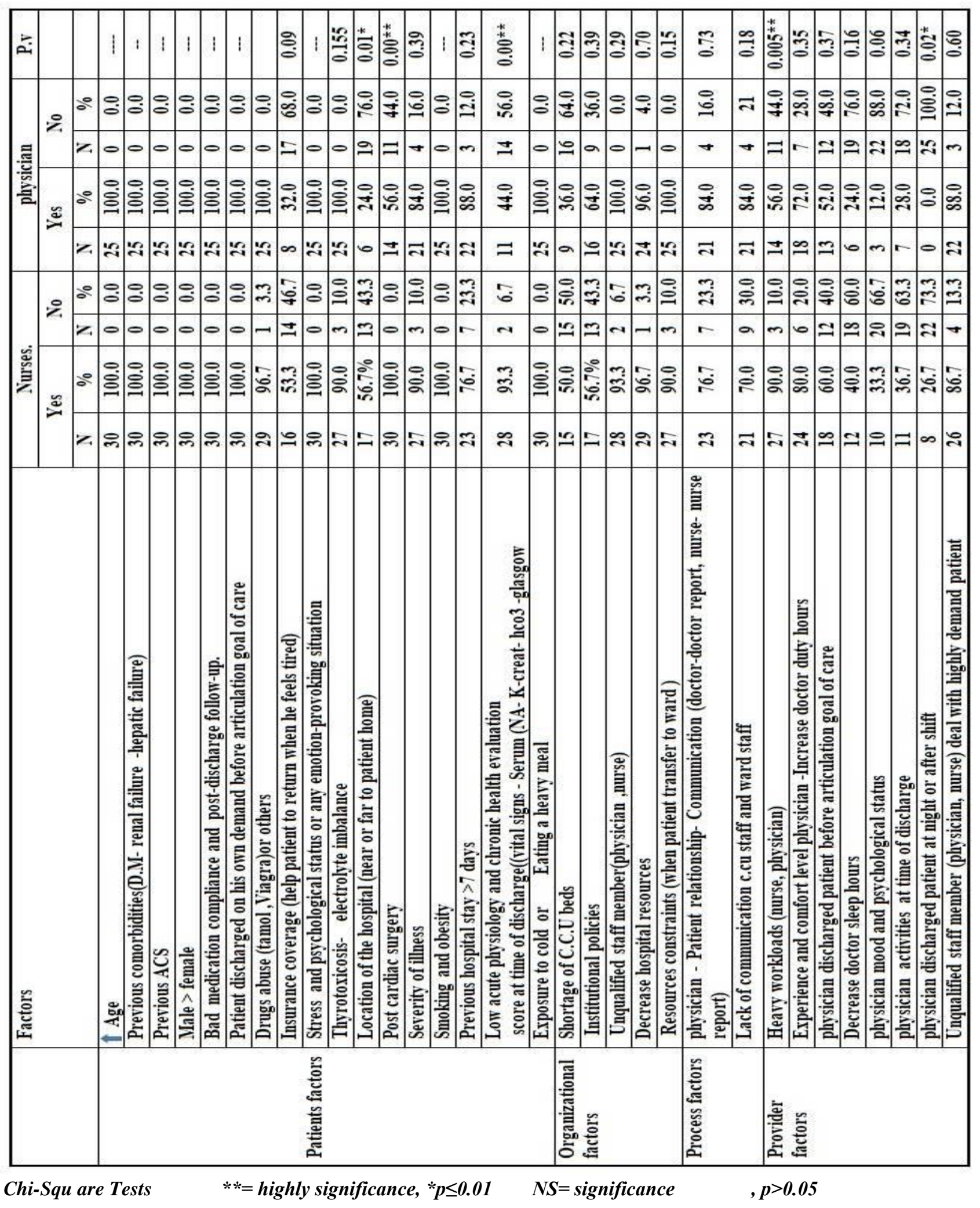


This Figure (1): Shows Distribution of demographic data for participant (doctors \& nurses), the results revealed that the nurse participated in this research were aged 25 years old, nearly half of nurses had 1-5 years of experience with $76.7 \%$ percentage and their level of education were intermediate ( Technical Institute ) with $73.3 \%$ percentage , and doctors were aged 33years old, half of the doctors had 5-10 years of experience with $52 \%$ and all of the doctor have higher level of education( bachelor degree) with $100 \%$ percentage.

This Table (1): Showed the patient factors that Comparison between the point of view for nurses and physician regarding patient. All nurses and physician agreed $(100 \%)$ that previous comorbidities (D.M.) and ACS in patients were contributing factors. Respondents also stressed the fact that bad psychological status, or any emotion-provoking situation were of significant factors as well almost all patient were male, Bad medication compliance and post-discharge follow-up- Drugs abuse (Tamol, Viagra) or others-Stress and psychological status or any emotion-provoking situation -Thyrotoxicosiselectrolyte imbalance-Post cardiac surgery -Severity of illness- Smoking and obesity) were contributing to patient readmission to coronary care unit.

As regarding Organizational factors all nurses and physician agreed $(100 \%)$ that Unqualified staff member (physician, nurse) were a risk factor of readmission .

Process factors as well, contributed to patient readmission to coronary care unit as reported by $100 \%$ of nurses and doctors in the form of (physician -Patient relationship- Communication (physician physician report, nurse- nurse report) -Lack of communication between CCU staff and ward staff were factors for readmission .

On the other hand, Provider factors contributing to patient readmission to the CCU as reported by $88.0 \%$ a majority of nurses and doctors was the presence of an unqualified staff member (physician, nurse) dealing with high demand patients

\section{Discussion}

Acutely ill patients are commonly found in general hospital units; some of who have been recently discharged from an intensive care unit (ICU). These patients may require a higher level of care than other ward patients and, due to the acuity of their illness, are at risk of readmission to ICU (Underwood, 2018).

Many studies indicated that patients readmitted to ICU have mortality rates up to six times higher than those not readmitted and are eleven times more likely to die in hospital. Several studies have retrospectively examined these readmissions but, despite this, there is still no clear indication of why ICU readmissions occur or what the common characteristics of readmitted patients are (Falsini et al., 2018)

The results of the present study presented as following :-

Regarding Sociodemographic characteristics

Based on the results of the present study; patients in coronary care unite (CCU) their mean age of patients were $60.03 \pm 9.45$ years. This agreed with Cameron et al., (2016) who found the mean age of CCU patients were more than 60 years old. However, Barry, \& Thompson, (2018) found that patents mean age was 67.5 (7.38). Prior research has shown that severity of illness is more important than age on patients' prognosis, and aggressive treatment strategies are not less cost-effective when provided to older patients (Schumacher et al., 2017). Regarding the point of view for nurses and physicians regarding factors of readmission:

The present study classified the factors into patients factors, Organizational factors, and process factors and Provider factors.

Regarding patients factors

Nearly all nurses and doctors agreed that increase patients Age, Previous comorbidities(D.M- renal failure hepatic failure, Previous ACS, Male > female, Bad medication compliance and post-discharge follow-up, Patient discharged on his own demand before articulation goal of care, Drugs abuse (tamol, Viagra)or others, Stress and psychological status or any emotion-provoking situation, Thyrotoxicosiselectrolyte imbalance, Post cardiac surgery, Severity of illness, Smoking and obesity, Previous hospital stay $>7$ days, Low APACHE score at time of discharge((vital signs - Serum (NA- K- creat- hco3 Glasgow, Exposure to cold or Eating a heavy meal, were the main patients factors of ICU readmission.

These findings are compatible with previous smaller studies Pignatiello et al., (2018) who identify the sick, elderly and chronically unwell patient, progressing slowly and in a tertiary hospital, to have greater odds of readmission. Patients with specific primary diagnoses on initial ICU admission also have greater odds of readmission.

(Ofoma, 2018) found that; the perceptions of physicians were mostly concordant with those of nursing staff, with the exception discharge decisionmaking, where physicians and nurses disagreed regarding the extent to which nursing opinion factored into decision-making by ICU physicians. Also, while physicians underscored the subjective nature of the discharge-making process as a cause of premature discharge from the ICU, nurses placed more emphasis on ICU occupancy pressures. 
With regards, (van Sluisveld. 2015 ) mentioned that the role of undefined goals of care, physicians were more attuned than nurses, to potential barriers that were usually encountered in adequately articulating these goals. Participants in this study perceived that the severity of illness and undefined goals of care were the predominant patient factors that predisposed to intensive care readmission. It follows logically that sicker patients are more likely to be readmitted.

The severity of illness both at the time of ICU admission and discharge is an independent risk factor for readmission (Wong et al., 2016).

In this respect Wilson et al., (2018) mentioned that; the comorbidities of a critically ill patient may not be easily modifiable. However,( Johnson et al., 2013) said that; decisions to limit life support are among the most important and difficult clinical decisions encountered by patients, families, and providers, with a host of factors contributing to physician variability in decisions to limit life support promptly and adequately articulate appropriate goals of care from the time of hospital admission to the time of index ICU discharge may inevitably lead to unnecessary and perhaps futile readmission to the $\mathrm{CCU}$ for certain categories of patients.

In a line of Process factors, the majority of the studied nurses and doctors agreed that Doctor-Patient relationship - Communication (doctor-doctor report, nurse- nurse report) and lack of communication CCU staff and ward staff. Ofoma et al., (2018) reported that effective communication improves clinical decision making and is essential for mitigating errors and achieving high-quality clinical outcomes. Poor communication among critical care teams is a contributing factor to adverse events including readmissions to intensive care.

The study of Yap et al., (2017) revealed that participants felt that a suboptimal atmosphere for efficient team communication often resulted in inadequacies and discrepancies in communicated content at times of team hand-off. Premature discharge from index ICU admission and other inefficiencies in transitioning patient care from hospital ICUs to general medical settings have been linked to unplanned ICU readmissions.

Traditionally, discharge decisions are made by attending physicians, in collaboration with other members of the ICU care team (Hartog et al., 2015). Nurses' reports of nurse-physician collaboration in decision-making at the time of been necessary .ICU discharge is positively associated with patient outcomes including ICU readmission and hospital mortality (Woo et al., 2017).

Adverse events from latent failures often arise from organizational factors that determine working conditions and institutional policies. In this regard, the present study nurses and doctors agreed that; institution lacked dedicated step-down units and participants cited this as a possible reason for readmissions that may not have

Implications Organizations may focus their efforts on modifying or improving aspects of non-patient factors that will improve outcomes for patients at risk of CCU readmission, for example by standardizing care processes and improving communication between multidisciplinary care teams. For instance, transitional care programs after discharge from intensive care have been shown to reduce the risk of ICU readmissions (Niven et al., 2014). Also, formalizing multidisciplinary input may improve CCU discharge decision-making, while additional training can improve the comfort level of care providers. Engaging institutional stakeholders can also raise awareness of the possible role of organizational factors (Stelfox et al., 2016).

Several risk stratification scores have been proposed as predictors of ICU readmission (Zheng et al., 2015). Predominantly composed of patient-centred factors, these scoring systems have, however, shown limited predictive abilities. The impact of non-patient system factors may explain the poor to modest discrimination and the inability of these models to reduce readmissions during real-world implementation (Badawi \& Breslow, 2012).

Ouanes et al.,2012 mentioned that there were future researches examining intensive care readmissions approached from a systems perspective should evaluate the role of non-patient factors and their relationship to patient factors .

\section{Conclusion}

Based on the results of the present study it can be concluded that

Nurses and physician perception about readmission cause that factors related to patients like age, comorbidities, medication bad adherence, Patient discharged on his own demand, and stress. Also, another factor related to the organization as unqualified staff members (doctor and nurse) and decrease hospital resources.

\section{Recommendations}

Based on the results of this study it was recommended that:

\section{- For patients :}

* Health education of the patients about factors that reduce readmission

* Provide written information to facilitate educating patients should be available and provided to every cardiac patient. 
For an organization

* Provide continuous training programs for all medical staff to improve the quality of care for all critically ill patients.

* Apply the students in CCU about how to deal with emergeny cases .

* Appropriate post-discharge follow up for complication early detection

- For research

* Repeat this research on large sample size and multi centres for generalization.

* Application of hospital-related readmission program to minimize the incidence of coronary diseases readmission rate.

\section{Reference}

1. Badawi O., Breslow M., (2012): zzz: (DELIRIUM CORDIS). European Heart Journal: Acute Cardiovascular Care, 7(7), 661-670.)

2. Danielle, A., Southern M., \& Galberth D., (2014): characterizing types of readmission after acute coronary syndrome hospitalization implication of quality reporting $\mathrm{J}$ Am Assoc. 2014:3:e $001046 \quad$ DOI 10.1161/JAHA.114.001046)

3. Evan. G., Ann, M., Doris, G, \&.Arabje, I., (2016): Association of Severity of Illness and Intensive Care Unit ASystemic Review Heart Lung .45(1)3-9

4. Elliott, M., \& Crookes, P., (2011): Readmission to intensive care: A qualitative analysis of nurses' perceptions and experiences HEART \& LUNG The Journal of Acute and Critical Care 40(4) 299309

5. Falsini, G., Grotti, S., Porto, I., Toccafondi, G., Fraticelli, A., Angioli, P., \& Romanelli, S., (2018): Long-term prognostic value of delirium in elderly patients with acute cardiac diseases admitted to two cardiac intensive care units: a prospective study

6. Hartog, C., Schwarzkopf, D., Riedemann, N., Pfeifer, R., Guenther, A., Egerland, K., \& Reinhart, K., (2015): End-of-life care in the intensive care unit: a patient-based questionnaire of intensive care unit staff perception and relatives' psychological response. Palliative medicine, 29(4), 336-345

7. ILai, Jab ChiehChang, SabTang, T., (2012): Readmission to the intensive care unit: A population-based approach Journal of the Formosan Medical Association 2012 111( 9), 504509-

8. Johnson D., Schmidt U., Bittner E., Christensen B., Levi R., Pino R., (2013): Delay of transfer from the intensive care unit: a prospective observational study of incidence, causes, and financial impact. Crit Care.;17(4): R128.

9. Niven D., Bastos J., Stelfox H., (2014): Critical care transition programs and the risk of readmission or death after discharge from an ICU: a systematic review and meta-analysis. Crit Care Med.;42(1):179-87.

10. Ofoma, U., Dong, Y., Gajic, O., \& Pickering, B., (2018): A qualitative exploration of the discharge process and factors predisposing to readmissions to the intensive care unit. BMC health services research, 18(1), 6

11. Ouanes, I., Schwebel C., Francais A., Bruel C., Philippart F., Vesin A., Soufir L., Adrie C., Garrouste-Orgeas M., Timsit J., ( 2012): A model to predict short-term death or readmission after intensive care unit discharge. J Crit Care. 2012;27(4): e421: 429.

12. Pignatiello, G., (2018): The Relationships Among Self-Regulation, Cognitive Load, Decision Support, and Decision Making Readiness in Surrogate Decision Makers for the Critically Ill (Doctoral dissertation, Case Western Reserve University, P.P. 35:48

13. Stelfox H., Bastos J., Niven D., Bagshaw S., Turin T., Gao S., (2016): Critical care transition programs and the risk of readmission or death after discharge from ICU. Intensive Care Med.;42(3):401-10.

14. Steven A., Victor T., Evan A., Leanne. H., Yenna S., Patricia, M., \& Michael J., Parr \& Ken M., (2010): Readmission to intensive care: development of a nomogram for individualising risk, Critical Care and Resuscitation - Vol (12) $\mathrm{No}(2) \cdot$ June 2010

15. Underwood, J., Ruane, S., \& Reynolds, K., (2018): Report on University Hospitals of Leicester NHS Trust Full Business Case for relocating Intensive Care Unit Level 3 beds out of Leicester General Hospital. DMU: Health Policy Research Unit., P.P.345:355

16. van Sluisveld N., Hesselink G., van der Hoeven J., Westert G., Wollersheim H., Zegers M., (2015): Improving clinical handover between intensive care unit and general ward professionals at intensive care unit discharge. Intensive Care Med.;41(4):589-604.

17. Wilson M., Rhudy L., Ballinger B., Tescher A., Pickering B., Gajic O., (2013): Factors that contribute to physician variability in decisions to limit life support in the ICU: a qualitative study. Intensive Care Med.;39(6):1009-18.

18. Wong, E., Parker, A., Leung, D., Brigham, E., \& Arbaje, A., (2016): Association of the severity of illness and intensive care unit 
readmission: A systematic review. Heart \& Lung, 45(1), 3-9

19. Woo, B., Lee, J., \& San Tam, W., (2017): The impact of the advanced practice nursing role on the quality of care, clinical outcomes, patient satisfaction, and cost in the emergency and critical care settings: a systematic review. Human resources for health, 15(1), 63

20. Yap, J., Abdul-Rahman, H., \& Chen, W., (2017): Collaborative model: Managing design changes with reusable project experiences through project learning and effective communication. International Journal of Project Management, 35(7), 1253-1271.

21. Yong, J., (2015): Readmission to Medical Intensive Care Units: Risk Factors and Prediction. (2015), Mar 1; 56(2): 543-549 Yonsei Med J published online 2015 Feb 9. doi: 10.3349/ymj.2015.56.2.543

22. Zheng, B., Zhang, J., Yoon, S., Lam, S., Khasawneh, M., \& Poranki, S., (2015): Predictive modelling of hospital readmissions using metaheuristics and data mining. Expert Systems with Applications, 42(20), 7110-7120. 